07,01

\title{
Динамическая модель упруго-пластического нормального столкновения сферической частицы с полупространством с учетом адгезионного взаимодействия в зоне контакта
}

\author{
(C) Я.А. Ляшенко ${ }^{1,2}$ \\ ${ }^{1}$ Берлинский технический университет, \\ Берлин, Германия \\ ${ }^{2}$ Сумский государственный университет, \\ Сумы, Украина \\ E-mail: i.liashenko@tu-berlin.de
}

(Поступила в Редакцию 20 августа 2018 г.

В окончательной редакции 29 августа 2018 г.)

\begin{abstract}
Численно решается задача столкновения сферической однородной частицы с полупространством. Рассчитан коэффициент восстановления нормальной скорости как функция начальной скорости при наличии диссипации механической энергии за счет пластических деформаций и адгезионного взаимодействия между поверхностями контактирующих тел. Проведен учет эффекта деформационного упрочнения, используя введенный ранее критерий нелокальной пластичности. Исследование проведено в безразмерных параметрах, что делает его универсальным. Найдено аналитическое выражение для критической начальной скорости, при превышении которой начинается пластическая деформация. Сравнение полученных в результате моделирования результатов с результатами имеющихся экспериментальных данных показывает хорошее соответствие.
\end{abstract}

Работа выполнена при финансовой поддержке Немецкого научно-исследовательского сообщества (DFG), проект РО 810-55-1.

DOI: 10.21883/FTT.2019.02.47130.240

\section{1. Введение}

В последние десятилетия активно развиваются теоретические и численные методы исследования контактных явлений твердых и вязко-упругих тел при различных условиях в зоне контакта. Это связано в частности c практической важностью понимания особенностей контактного взаимодействия в промышленности при проектировании различных технологических процессов и оборудования. Одно из активно разрабатываемых на сегодняшний день направлений в контактной механике связано с исследованием динамического процесса соударения твердых тел. Указанные исследования проводятся в том числе и для описания особенностей динамического поведения гранулированных сред [1-3]. Как правило, конечной целью является оптимизация разнообразных технологических процессов (сушка и фасовка удобрений, приготовление гранулированных кормов, гранулированного твердого топлива из отходов деревообрабатывающей промышленности, гранулированных медицинских препаратов и т.п.). Вторым важным направлением, которое использует достижения физики столкновений, является моделирование природных явлений, сюда входят такие явления как землетрясения, перемещения ледников, оползни, формирование горных систем, и т.п. Более того, контактные явления оказывают существенное влияние при формировании астероидов и планетарных систем [4], а также при механических манипуляциях с наноразмерными систе- мами [5], что в связи с современными тенденциями миниатюризации механических устройств имеет большую перспективу практического применения. Стоит отметить, что в общем случае описание контактных явлений представляет сложную задачу, поскольку здесь существенную роль играют адгезионные силы $[5,6]$, шероховатости, наличие пластической деформации $[7,8]$, наличие третьего тела в зоне контакта, и многие другие особенности.

Процессы, происходящие при столкновениях твердых частиц, изучаются также и экспериментально. Например, в работах $[9,10]$ теоретически и экспериментально изучается отражение стального шарика от стеклянной пластины с учетом диссипации, связанной с распространением упругих волн в материале пластины. Авторы изучают влияние толщины пластины (которая в предлагаемой нами статье считается бесконечной, поскольку мы работаем в пределе полупространства), радиуса шара, а также начальной скорости шарика. В другой работе [11] дополнительно изучается канал диссипации энергии, связанный с вязко-упругими процессами, проходящими в зоне контакта. В [12] в ходе компьютерного эксперимента изучается столкновение упруго-пластического шара с несжимаемым полупространством, причем в указанной работе проведен учет эффекта деформационного упрочнения материала. В работе [13] построена динамическая модель упругопластического столкновения сферических частиц, которая показывает удовлетворительное совпадение с из- 
вестными экспериментальными данными других авторов. Достоинством указанных работ несомненно является то, что авторы не только проводят эксперимент (либо компьютерное моделирование), но и строят соответствующую теорию, которая в достаточной мере его объясняет, или же используют имеющиеся в литературе теоретические подходы для объяснения результатов эксперимента. Краткое описание существующих на сегодняшний день теорий столкновений, а также некоторых экспериментов можно найти в недавно вышедшем обзоре [14]. Однако, несмотря на изобилие теоретических и экспериментальных работ, вопрос физики столкновений остается открытым, поскольку, как это показано, например, в работе [15], различные подходы зачастую дают весьма противоречивые результаты. Более того, результаты экспериментов также существенно разнятся, а связано это с различными типами контактного взаимодействия. В частности, с различными механизмами протекания пластической деформации. В предлагаемой статье мы рассматриваем один из них. Существуют также работы, в которых изучаются специфические условия столкновения. К примеру, в [16] рассматривается столкновение упругой частицы с упругой пластиной, на которую нанесен тонкий слой вязкой жидкости, что приводит к возникновению дополнительного канала диссипации механической энергии. Подобная ситуация изучалась ранее в [17].

В предыдущей работе [18] мы построили модель нормального столкновения сферической частицы с полупространством, используя введенный ранее в [19] критерий нелокальной пластичности. Такая модель позволила нам довольно точно описать результаты эксперимента, проведенного в работе [20]. Однако, в [18] описывается только один эксперимент и модель оперирует параметрами этого эксперимента. Полученное с высокой точностью совпадение с имеющимися экспериментальными данными доказывает адекватность построенной модели, однако за конкретными параметрами из виду теряется универсальность предложенного нами подхода, который способен описывать столкновения при наличии пластических деформаций в широком диапазоне параметров. Настоящая работа предпринята с целью универсализации динамической модели, предложенной в [18]. В отличии от работы [18] здесь исследование проведено в безразмерных параметрах, при этом удалось получить семейство кривых, которые описывают столкновения во всем диапазоне возможных параметров эксперимента. Однако, такая модель позволяет описать только те ситуации, в которых справедливым является критерий пластичности, предложенный в работе [19]. Стоит понимать, что пластическое деформирование является довольно сложным процессом и здесь возможно множество различных сценариев поведения, которые не укладываются в рамки построенной модели. Однако в тех ситуациях, в которых справедлив критерий пластичности [19], результаты экспериментов при их описании в рамках предлагаемого нами подхода воспроизводятся с высокой точностью.

\section{2. Формулировка модели пластической деформации при нормальном индентировании параболического индентора в упругое полупространство}

Одним из перспективных методов исследования механики столкновений является метод редукции размерности (MDR) [21]. В рамках этого метода было исследовано столкновение частиц без проскальзывания в зоне контакта [22], с конечным коэффициентом трения [23], а также при наличии сил адгезии между поверхностями контактирующих тел [24].

B рамках MDR в первую очередь необходимо заменить оригинальный трехмерный профиль контактирующего с полупространством тела $z=f(r)$ на его одномерный эквивалент $g(x)$ согласно правилу $[21,25]$

$$
g(x)=|x| \int_{0}^{|x|} \frac{f^{\prime}(r)}{\sqrt{x^{2}-r^{2}}} \mathrm{~d} r .
$$

Для сферического индентора $f(r)=r^{2} / 2 R$ имеем зависимость $g(x)=x^{2} / R$. Далее полупространство и взаимодействующее с ним тело одновременно заменяются на упругое основание, состоящее из последовательности невзаимодействующих пружин с нормальной жесткостью [25]

$$
k_{z}=E^{*} \Delta x
$$

где $\Delta x-$ величина дискретизации (расстояние между пружинами). Эффективный модуль упругости определяется как

$$
\frac{1}{E^{*}}=\frac{1-v_{1}^{2}}{E_{1}}+\frac{1-v_{2}^{2}}{E_{2}},
$$

где $E_{1,2}$ - модули упругости, а $v_{1,2}-$ коэффициенты Пуассона индентора и полупространства.

После этого можно приступать непосредственно к моделированию контактных явлений. Для этого производится вдавливание индентора с профилем $g(x)$ в упругое основание на глубину $d$, в результате чего индивидуальные пружины приобретают смещения

$$
u_{z}(x)=d-g(x)=d-\frac{x^{2}}{R} .
$$

Радиус контакта $a$ определяется условием $u_{z}(a)=0$, откуда имеем [26]

$$
a=\sqrt{R d} .
$$

Чтобы определить действующую нормальную силу $F_{z}$, необходимо сложить силы натяжения от каждой пружины в контакте

$$
F_{z}=E^{*} \int_{-a}^{a} u_{z}(x) \mathrm{d} x=2 E^{*} \int_{0}^{\sqrt{R d}}\left(d-\frac{x^{2}}{R}\right) \mathrm{d} x=\frac{4 E^{*} \sqrt{R d^{3}}}{3} .
$$


При численном моделировании будем пользоваться дискретным аналогом интеграла (6)

$$
F_{z}=E^{*} \Delta x \sum_{\text {cont }} u_{z}\left(x_{i}\right)
$$

где суммирование производится по всему контакту.

В работе [19] был предложен критерий нелокальной пластичности, который легко имплементировать в MDR. B [19] рассматривается случай, когда теряет смысл критическое напряжение текучести материала. Такая ситуация неизбежно наблюдается для малых радиусов контакта. В указанной ситуации „твердость“ зависит от размера контакта. Согласно [19], действующая в контакте нормальная сила может быть записана в виде

$$
F_{z}=\pi q_{c} a
$$

где $a-$ радиус контакта, а $q_{c}$ имеет смысл критической линейной плотности силы, при превышении которой начинается пластическая деформация. Критическая сила $f_{c}$, действующая на одну пружину, определится выражением

$$
f_{c}=\frac{F_{z}}{2 a / \Delta x} .
$$

Из (8) и (9) найдем величину критической силы $f_{c}[19]$

$$
f_{c}=\frac{\pi}{2} q_{c} \Delta x
$$

при превышении которой начинается пластическое деформирование выделенной пружины. После начала процесса пластического деформирования при дальнейшей деформации пружины упругая сила равна $f_{c}(10)$ и не изменяется, а пластическая деформация при этом накапливается.

На рис. 1 для большей наглядности показана хорошо известная классическая схема нагружения индивидуальной пружины, предполагающая идеальную пластичность без эффекта упрочнения. Здесь до критического значения $f_{c}(10)$ пружина деформируется упруго. Это упругий участок зависимости, на котором нормальная сила задается законом Гука

$$
F_{z}\left(x_{i}\right)=\left[u_{z}\left(x_{i}\right)-u_{z}^{p l}\left(x_{i}\right)\right] E^{*} \Delta x,
$$

в котором для рассматриваемого упругого участка $u_{z}^{p l}\left(x_{i}\right)=0$. На упругом участке при разгружении пружины она полностью восстанавливает свою первоначальную форму. Если же натяжение пружины превысит критическое значение

$$
u_{z}\left(x_{i}\right)>u_{z}^{\text {crit }}=\frac{f_{c}}{E^{*} \Delta x}
$$

то при дальнейшем нагружении величина упругой силы становится тождественно равной критическому значению $F_{z}\left(x_{i}\right)=f_{c}$. Разгружение пружины, которая пластически деформирована, происходит также по гуковскому

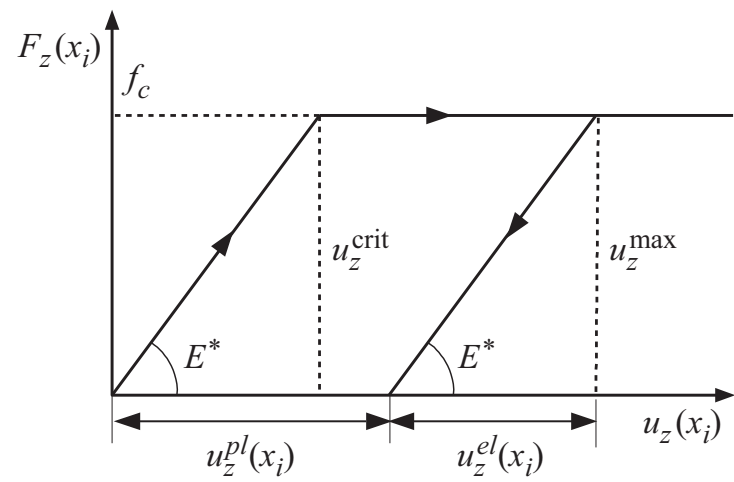

Рис. 1. Схематический вид кривой нагружения для выделенной пружины с координатой $x_{i}$.

участку (см. рис. 1), при этом пластическая составляющая смещения пружины может быть найдена как

$$
u_{z}^{p l}\left(x_{i}\right)=u_{z}^{\max }-u_{z}^{\text {crit }} .
$$

В процессе разгружения пружины нормальную силу следует вычислять по формуле (11), где при наличии пластической деформации $u_{z}^{p l}\left(x_{i}\right) \neq 0$.

При таким образом введенном критерии пластичности сначала будут деформироваться пружины, имеющую максимальную длину, когда глубина индентирования превысит критическую величину $d_{c}$. Это значение легко определить, если приравнять критическую силу $f_{c}(10)$ к упругой силе, создаваемой центральной пружиной, натяжение которой определяется формулой (4) при $x=0$ (в центре контакта) и совпадает с критической глубиной индентирования $d_{c}$. Упругая сила натянутой пружины определяется как $F=k_{z} d_{c}$, где нормальная жесткость $k_{z}$ задается формулой (2). Фактически при этом мы находим критическую величину натяжения пружины (12), где $u_{z}^{\text {crit }} \equiv d_{c}$. Отсюда имеем критическую глубину индентирования

$$
d_{c}=\frac{\pi q_{c}}{2 E^{*}} .
$$

При $d>d_{c}$ (14) начинается пластическое деформирование, если $d<d_{c}$, мы находимся в чисто упругой области.

На рис. 2 показана схема, показывающая индентирование жесткого профиля $g(x)$ в упруго-пластическое основание. Здесь показан радиус контакта $a$ (5). Общая глубина индентирования обозначена как и ранее буквой $d$. Критическая величина $d_{c}(14)$ определяет радиус области пластической деформации $b$. Поскольку мы используем идеализированную модель пластического течения, показанную на рис. 2 , в области $-b<x<b$ все пружины дают эквивалентный вклад в упругую силу, определяемый выражением (10). В областях контакта $b<x<a$ и $-a<x<-b$ пружины деформированы упруго, а их вклад в нормальную силу определяется законом Гука (11). Используя (14) и выражение для радиуса контакта (5) легко найти критическое значение радиуса, при котором начинается пластическая 


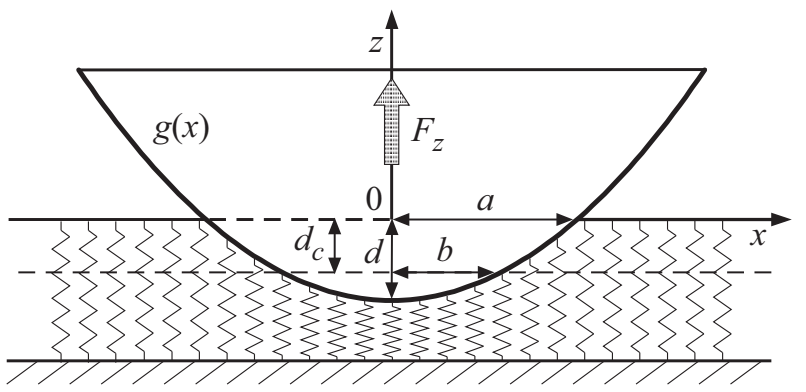

Рис. 2. Схема упруго-пластически деформированного основания.

деформация

$$
a_{c}=\sqrt{\frac{\pi R q_{c}}{2 E^{*}}} .
$$

В случае отсутствия пластических деформаций сила, действующая на индентор со стороны полупространства, определяется записанным выше выражением (6). Однако при наличии пластического деформирования центральные пружины, находящиеся в области $-b<x<b$, показанной на рис. 2, будут давать меньший вклад в упругую силу. Для определения этой силы необходимо найти величину радиуса $b$, которая исходя из геометрических соображений (используем рис. 2 и выражения (4), (14)) определится как

$$
b=\sqrt{R d-\frac{\pi R q_{c}}{2 E^{*}}} .
$$

Отметим, что в ситуации, когда полупространство не обладает упругими свойствами и пластические деформации наступают в самом начале контакта $\left(q_{c}=0\right)$, согласно (5) выражение (16) дает $b \equiv a$. Вклад в упругую силу от пружин в области $-b<x<b$ может быть определен как количество пружин в этой зоне $2 b / \Delta x$, умноженное на критическую силу $f_{c}(10)$, поскольку согласно используемому критерию пластичности все пружины в этой зоне дают одинаковый вклад, равный $f_{c}$. Вклады от упруго натянутых пружин могут быть как и ранее найдены по формуле (6), однако с измененными пределами интегрирования, поскольку упругие области теперь задаются двумя симметричными относительно начала координат интервалами $-a<x<-b$ и $b<x<a$. В итоге будем иметь

$$
F_{z(e l, p l)}=2 E^{*} \int_{b}^{a}\left(d-\frac{x^{2}}{R}\right) \mathrm{d} x+\pi q_{c} b
$$

что с учетом определений (5) и (16) дает окончательное выражение

$F_{z(e l, p l)}=\frac{4 E^{*} \sqrt{R d^{3}}}{3}-\frac{H\left(2 E^{*} d-\pi q_{c}\right)}{3} \sqrt{\frac{2 R\left|2 E^{*} d-\pi q_{c}\right|^{3}}{E^{*}}}$,

где $H(x)$ - функция Хевисайда. Выражение (18) отличается от (6) наличием второго слагаемого. Функция Хе- висайда в (18) введена для того, чтобы описать переход от чисто упругой деформации (когда в (18) существует только первое слагаемое) к наличию пластической деформации в случае $2 E^{*} d-\pi q_{c}>0$. Для этой же цели в подкоренном выражении указанная разность взята по абсолютному значению, поскольку при отсутствии пластической деформации подкоренное выражение во втором слагаемом в (18) становится отрицательным. Легко видеть, что в случае $q_{c}=0$ выражение (18) дает значение $F_{z(e l, p l)}=0$, то есть упругая сила при этом, как уже указывалось выше, полностью отсутствует.

Таким образом, при чисто упругом индентировании справедлива формула (6). В случае, когда по всей области контакта наблюдается пластическая деформация, действует сила (8). При дальнейшем моделировании будем использовать выражение (18). Это выражение получено непосредственно с использованием критерия пластичности (10) и соответствует ситуации, когда пластически деформируются центральные пружины в контакте, для которых этот критерий выполняется, а на краях контакта пружины деформируются упруго. Приведем зависимости этих трех сил от глубины индентирования. Для универсальности представления результатов далее везде будем использовать безразмерные переменные. Все силы измерим в единицах критической силы $f_{c}$, которая численно равна упругой силе (6) в момент, когда начинается пластическая деформация (глубина индентирования $d$ при этом тождественно равна критическому значению $\left.d_{c}(14)\right)$

$$
F_{c}=\frac{2 \pi q_{c}}{3} \sqrt{\frac{\pi R q_{c}}{2 E^{*}}} .
$$

На рис. 3 показаны зависимости сил (6), (8) и (18) от глубины индентирования $d$.

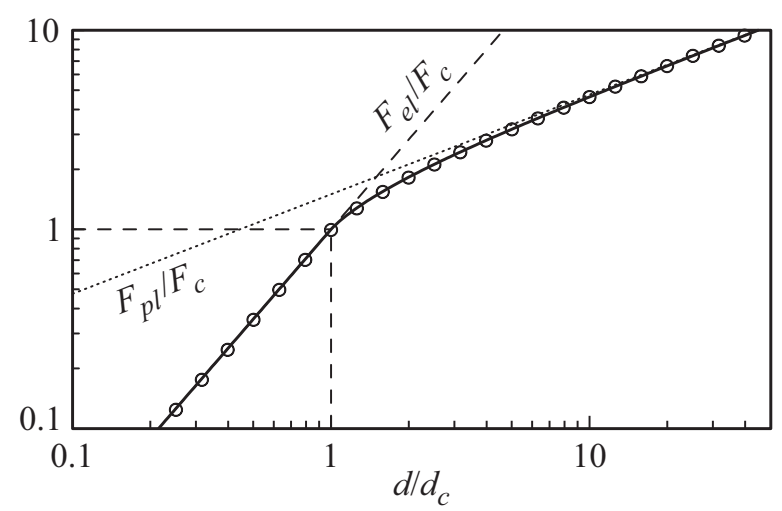

Рис. 3. Зависимости сил $F_{e l}(6)$ при чисто упругом индентировании (штриховая прямая), $F_{p l}(8)$ при наличии пластической деформации по всей области контакта (пунктирная прямая) и силы $F_{(e l, p l)}(18)$, которая следует из критерия пластической деформации (10) (сплошная кривая + кружки) от безразмерной глубины индентирования $d / d_{c}$. Все приведенные на рисунке силы измерены в единицах $F_{c}(19)$. Сплошная кривая получена по формуле (18), кружки - результат моделирования согласно описанной выше процедуре (1)-(13). 
Из рисунка следует, что в области $d / d_{c}<1$ сила (18) совпадает с упругой силой (6), поскольку пластическая деформация полностью отсутствует. По мере расширения контакта и увеличения контактного радиуса (с ростом глубины индентирования $d$ ) выражение (18) приближается к зависимости, задаваемой формулой (8). Однако при этом (8) является асимптотикой, поскольку при использовании критерия пластичности (10) всегда существуют области на краю контакта, которые деформируются упруго.

Штриховая (формула (6)) и пунктирная (формула (8)) линии на рис. 3 в безразмерном виде задаются уравнениями

$$
\frac{F_{e l}}{F_{c}}=\left(\frac{d}{d_{c}}\right)^{3 / 2}, \quad \frac{F_{p l}}{F_{c}}=\frac{3}{2}\left(\frac{d}{d_{c}}\right)^{1 / 2} .
$$

Последние два уравнения представляют собой степенные функции, поэтому на рис. 3 в двойных логарифмических координатах они являются прямыми линиями. Согласно рисунку эти прямые пересекаются в точке $d / d_{c}=3 / 2$ и $F_{e l} / F_{c}=F_{p l} / F_{c}=3 \sqrt{6} / 4$, которую условно можно считать точкой перехода режима упругой деформации в режим деформации пластической. Хотя, как это видно из рисунка, определенной точки перехода между этими режимами нет и такой переход осуществляется плавно.

\section{3. Моделирование динамики нормального столкновения однородной сферической частицы с упругим полупространством}

Процесс нормального столкновения однородного сферического тела с упругим полупространством для удобства можно разбить на две фазы. Во время первой фазы происходит погружение шарика в полупространство. В процессе погружения (индентирования) нормальная скорость движения шарика будет уменьшаться вплоть до нулевого значения, а его кинетическая энергия переходить в упругую энергию сжатых пружин. В рамках постановки задачи часть энергии затрачивается на работу пластической деформации. Вторая фаза начинается в момент, когда нормальная скорость переходит через нулевое значение и изменяет знак, то есть фаза представляет непосредственно процесс отскока шарика. Во второй фазе скорость шарика увеличивается от нуля до значения, при котором исчезает контакт между шариком и полупространством. Отношение этой скорости (взятой с обратным знаком) к начальной называется коэффициентом восстановления нормальной скорости. Для нашего моделирования важно, что в таким образом выделенных фазах столкновения шарик всегда движется в противоположных направлениях. Это означает, что для каждой пружины, показанной на рис. 2, будет сначала осуществляться нагружение с монотонно увеличивающимся значением $u_{z}\left(x_{i}\right)$, а при разгружении величина $u_{z}\left(x_{i}\right)$ будет монотонно уменьшаться.
Рассмотрим непосредственно процесс столкновения сферы с полупространством. Как и выше будем обозначать начальную скорость шарика $v_{z 0}$. Мы рассматриваем упрощенную ситуацию, в которой шарик не вращается (начальная циклическая частота равна нулю), поэтому тангенциальное движение отсутствует, а векторы начальной скорости и скорости отскока противоположно направлены и коллинеарны. При отлете от поверхности циклическая частота вращения шарика также равна нулю. Одной из основных величин в таких экспериментах является коэффициент восстановления нормальной скорости $e_{n}$

$$
e_{n}=-\frac{v_{z}}{v_{z 0}}
$$

где $v_{z}$ - скорость шарика, с которой он отлетает от поверхности полупространства. Поскольку скорости $v_{z}$ и $v_{z 0}$ разнонаправленны и имеют противоположные знаки, значение $e_{n}>0$. Для определения зависимости скорости шарика $v_{z}$ от времени необходимо решить единственное уравнение движения

$$
m \dot{v}_{z}=-F_{z}
$$

где нормальная сила $F_{z}$ при контакте шарика с поверхностью согласно выражению (7) всегда положительна. Решая уравнение (22) с использованием описанной выше процедуры, можно найти конечную скорость шарика (при его отлете от поверхности полупространства), а значит и величину коэффициента восстановления $e_{n}(21)$.

Существует большое количество работ, в которых изучаются особенности столкновений твердых тел при наличии пластической деформации. В частности, в $[8,27]$ показано, что при столкновении сферического тела с упругим полупространством пластические эффекты начинают проявляться, если нормальная скорость превышает критическое значение

$$
\nu_{z 0}^{\text {crit }}=\left(\frac{\pi}{2 E^{*}}\right)^{2}\left(\frac{2}{5 \rho}\right)^{1 / 2}\left(\frac{2 E^{*} a_{c}}{\pi R}\right)^{5 / 2} .
$$

Здесь величина $E^{*}$ определяется равенством (3), $a_{c}$ контактный радиус, при котором начинается пластическое течение, а $\rho$ - плотность материала, из которого изготовлен шар, соударяющийся с полупространством. В рассматриваемом нами случае нелокальной пластичности критический радиус определяется введенным ранее соотношением (15).

В работе [8] коэффициент восстановления $e_{p}$, где кинетическая энергия теряется только за счет пластических деформаций, был определен как

$$
\begin{aligned}
e_{p}= & \left(\frac{6 \sqrt{3}}{5}\right)^{1 / 2}\left[1-\frac{1}{6}\left(\frac{v_{z 0}^{\text {crit }}}{v_{z 0}}\right)^{2}\right]^{1 / 2} \\
& \times\left[1+2 \sqrt{\frac{6}{5}\left(\frac{v_{z 0}}{v_{z 0}^{\text {crit }}}\right)^{2}-\frac{1}{5}}\right]^{-1 / 4} .
\end{aligned}
$$


Однако в предлагаемой работе мы рассматриваем случай нелокальной пластической деформации, когда твердость является функцией размера контакта [19]. Поэтому в нашем случае выражение (24) не верно и для определения коэффициента восстановления необходимо проводить численное моделирование. Но при этом будем определять скорость $v_{z 0}^{\text {crit }}$ также по формуле (23) и в процессе моделирования выясним, от каких параметров зависит коэффициент восстановления $e_{p}$ в нашем случае. После подстановки в (23) выражения для критического радиуса $a_{c}$ (15) будем иметь значение критической скорости в виде

$$
v_{z 0}^{\text {crit }}=\frac{2^{-1 / 4}}{\sqrt{5 \rho}}\left(\frac{q_{c}}{R}\right)^{5 / 4}\left(\frac{\pi}{E^{*}}\right)^{3 / 4} .
$$

Выражение (25) используется далее для представления результатов численного моделирования. На рис. 4 сплошной кривой показана зависимость коэффициента восстановления $e_{p}$ от безразмерной скорости, которая определяется формулой (24), полученной в работе [8]. Звездочками (нижняя кривая) здесь обозначена аналогичная зависимость, полученная в результате численного моделирования при использовании критерия пластичности (10). Численно рассчитанная кривая состоит из символов, каждый из которых получен при различном наборе параметров, в которые входят плотность материала шарика $\rho$, эффективный модуль упругости $E^{*}(3)$, радиус сферы $R$, а также параметр $q_{c}$. При моделировании для каждой последующей расчетной точки постепенно увеличивалась начальная скорость шарика $v_{z}$, и для каждого значения скорости (расчетной точки на графике) все указанные выше параметры выбирались произвольным образом. После этого значение $q_{c}$ вычислялось по формуле (25) таким образом, чтобы получить следующую точку на графике (на рис. 4 расстояния между любыми двумя соседними точками по оси абсцисс в логарифмическом масштабе одинаковы). Таким образом, каждая точка на графике соответствует произвольному набору параметров и начальных условий, однако вместе эти точки дают единую зависимость. Это говорит о том, что как и в случае, описываемом формулой (24), результирующий коэффициент восстановления зависит только от начальной нормальной скорости, нормированной на величину критической скорости (25), при которой начинаются пластические деформации.

Стоит отметить, что из рис. 4 следует, что в обоих случаях (сплошная кривая и звездочки) коэффициент восстановления начинает уменьшаться от единичного значения (отсутствие пластической деформации), когда начальная скорость превышает критические значение $v_{z 0}^{\text {crit }}$. Причем в случае аналитического результата (формула (24)) и проведенного моделирования эти скорости представляют различные значения, поскольку используемая в моделировании $v_{z 0}^{\text {crit }}(25)$ получена исходя из критерия пластичности (10), а изначальное выражение (23) получено исходя из других соображений. Это объясняет

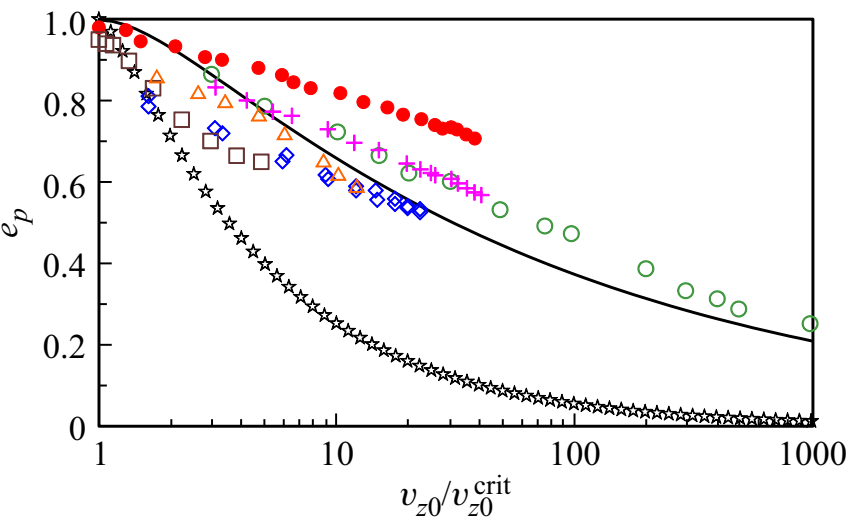

Рис. 4. Коэффициент восстановления нормальной скорости $e_{p}$, рассчитанный по аналитической формуле (24) (сплошная кривая) и полученный в результате численного моделирования (нижняя кривая, показанная звездочками). Символами показаны результаты экспериментов различных авторов (см. описание далее в тексте).

тот факт, что приведенные на рисунке кривые так сильно отличаются.

На рис. 4 также показаны результаты некоторых экспериментов различных авторов. Ромбами показаны результаты экспериментальной работы [28], в которой исследовалось столкновение двух шариков, выполненных из стали, используемой для изготовления подшипников (диаметр шариков при этом составлял $25.4 \mathrm{~mm}$ ). Треугольниками показаны результаты, описанные в работе [13], которые соответствуют отражению шарика, выполненного из амония флюоресцеина диаметром $4.4 \mu \mathrm{m}$, при его отражении от силиконовой мишени. Пустые кружки - это результаты компьютерного моделирования в рамках метода конечных элементов [12]. Крестиками показаны результаты работы [29], где шарик из алюминиевого оксида диаметром $5 \mathrm{~mm}$ отражался от пластины, выполненной из алюминиевого сплава. Заполненные кружки (верхние символы на рисунке) это результаты полностью аналогичного эксперимента по отражению шарика от стальной пластины [29]. И наконец квадратами на рисунке показаны результаты эксперимента, проведенного в работе [20], где изучалось отражение стального шарика диаметром $1.27 \mathrm{~cm}$ от стальной пластины. Здесь мы привели результаты лишь нескольких работ, на самом деле на сегодняшний день их гораздо больше.

Из рис. 4 видно, что большинство экспериментальных результатов удовлетворительно описывается теоретической кривой (24) и при этом все они очень далеки от кривой (кроме разве что показанных квадратами), которая получена в рамках предлагаемой нами численной модели (нижняя кривая, звездочки). Это связано в первую очередь с тем, что полученная нами кривая предполагает реализацию идеальной пластичности (см. рис. 1), а такая ситуация очень редко реализуется в природе, и для этого нужны специфические материалы 
и условия эксперимента. Поэтому для описания реально наблюдаемых процессов при столкновении необходимо учитывать эффект деформационного упрочнения (см. следующий раздел статьи). Однако стоит также отметить и тот факт, что кривая (24) описывает не все эксперименты, результаты которых показаны на рис. 4. Это не делает модель (24) универсальной, поэтому даже сегодня интенсивно проводятся новые эксперименты и на их основе строятся новые теории. В нашем случае мы используем критерий нелокальной пластичности (10), который ранее для описания процесса столкновений не использовался (не считая нашу предыдущую работу [18]). Этот критерий справедлив не для всех ситуаций и условий, как это описано выше, поэтому предлагаемая нами модель также является частным случаем.

\section{4. Учет эффекта деформационного упрочнения}

Ранее в работе [18] нами уже проводилось моделирование нормального столкновения с использованием критерия пластичности (10). А именно, мы теоретически описали эксперимент, проведенный в работе [20]. В этой работе экспериментально определялась зависимость коэффициента восстановления нормальной скорости $e_{n}$ (21) от значения начальной нормальной скорости шарика $v_{z 0}$. А именно, изучалось отражение шарика от гладкой стальной пластины, которую мы при моделировании считали полупространством. Поскольку в реальных экспериментах всегда присутствуют неровности поверхностей, которые деформируются при ударе, незначительная диссипация энергии присутствует даже в области упругого удара при $v_{z 0}<v_{z 0}^{\text {crit }}(23)$. Более того, к диссипации приводит и трение при движении в воздушной среде (эксперимент в [20] проводился в воздухе). Авторам работы [20] после тщательной полировки поверхностей контактирующих объектов удалось достичь максимального значения коэффициента восстановления в упругой области $e_{n} \approx 0.95$, а это очень близко к 1 , что говорит о высокой точности проведенных измерений.

Предлагаемая нами модель упруго-пластического столкновения предполагает идеально гладкие поверхности контактирующих тел, и в этом случае при малых скоростях нормального соударения (когда реализуется чисто упругое столкновение) коэффициент восстановления $e_{n}=1$. Однако, как мы это указали выше, за счет влияния факторов, которые не учитываются в нашей модели, в работе [20] максимальное значение коэффициента восстановления нормальной скорости не превышало значение 0.95. Поэтому при расчетах в [18] мы производили нормировку коэффициента восстановления на это максимальное значение.

Отметим, что практически во всех реальных ситуациях при пластическом деформировании происходит деформационное упрочнение материала в зоне пластического течения. Как показано нами в работе [18], экспериментальные результаты из [20] могут быть описаны в

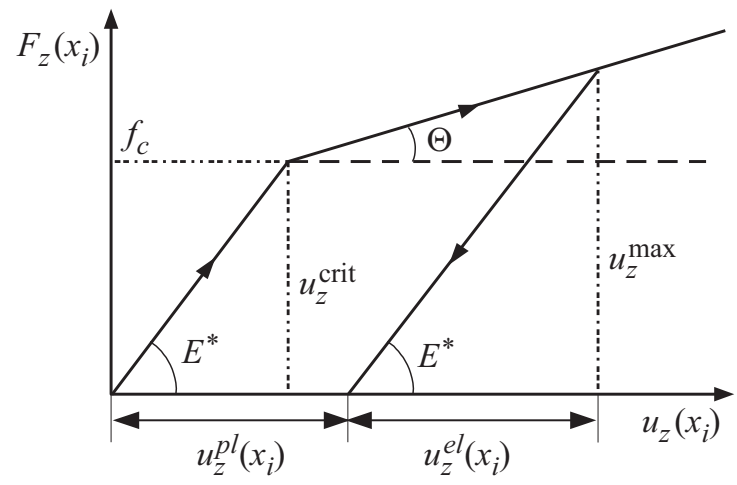

Рис. 5. Схематический вид кривой нагружения для выделенной пружины с координатой $x_{i}$ с деформационным упрочнением материала.

рамках предлагаемой модели только при учете деформационного упрочнения материала. При учете упрочнения диаграмма нагружения имеет вид (ср. с рис. 1), показанный на рис. 5 [30]. Здесь отличие от диаграммы, показанной на рис. 1, состоит в том, что при превышении упругой силой критического значения $f_{c}$ дальнейшее деформирование также происходит согласно закону Гука, но с более пологим наклоном, который задается коэффициентом упрочнения $\Theta$. Разгружение, как это показано на рисунке, происходит по гуковскому участку с наклоном, задающимся эффективным модулем упругости $E^{*}$.

Введенные ранее формулы (11) - (13) для учета упрочнения необходимо преобразовать [18]. Итак, до критического значения $f_{c}(10)$ пружина деформируется упруго, а нормальная сила задается законом Гука (11), в котором $u_{z}^{p l}\left(x_{i}\right)=0$. На упругом участке при разгружении пружины она полностью восстанавливает свою первоначальную форму. Поэтому если в процессе соударения начальная скорость шарика меньше критической и пластические деформации не наблюдаются, коэффициент восстановления равен 1, поскольку диссипация энергии отсутствует. Если же натяжение пружины превысит критическое значение $u_{z}\left(x_{i}\right)>u_{z}^{\text {crit }}(12)$, то величина упругой силы $F_{z}\left(x_{i}\right)$ задается соотношением (без учета упрочнения ранее использовалось соотношение $F_{z}\left(x_{i}\right)=f_{c}$, которое следует из (26) при $\Theta=0$ )

$$
F_{z}\left(x_{i}\right)=f_{c}+\left[u_{z}\left(x_{i}\right)-u_{z}^{\text {crit }}\right] \Theta \Delta x .
$$

При разгружении пластически деформированной пружины по гуковскому участку (см. рис. 5) пластическая составляющая смещения пружины может быть найдена по формуле (ср. с (13))

$$
u_{z}^{p l}\left(x_{i}\right)=\left(u_{z}^{\max }-u_{z}^{\text {crit }}\right)\left(1-\frac{\Theta}{E^{*}}\right) .
$$

В процессе разгружения пружины нормальную силу следует вычислять по формуле $(11)$, где $u_{z}^{p l}\left(x_{i}\right)$ определяется согласно (27). 


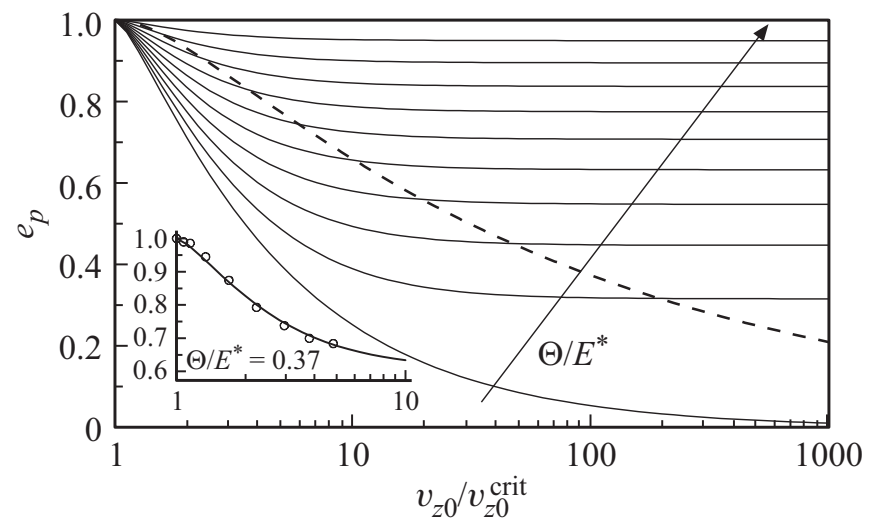

Рис. 6. Зависимости коэффициента восстановления нормальной скорости $e_{p}(21)$ как функции нормированного значения начальной скорости шарика $v_{z 0} / v_{z 0}^{\text {crit }}$. Кривые, расположенные снизу вверх, построены при значениях $\Theta / E^{*}$ от 0 до 0.9 с шагом 0.1. Увеличение параметра $\Theta / E^{*}$ показано стрелкой. На вставке к рисунку приведена кривая для значения $\Theta / E^{*}=0.37$, на которую наложены данные эксперимента (кружки), из работы [20], нормированные на максимальное наблюдаемое экспериментально значение $e_{p}=0.95$. Пунктирной линией показано решение (24).

Таким образом, в рамках описанной процедуры (11), (26) и (27) дополнительно появляется безразмерный параметр $\Theta / E^{*}$, значение которого влияет на коэффициент восстановления нормальной скорости. При $\Theta / E^{*}=0$ имеем ситуацию, показанную звездочками на рис. 4. В другом предельном случае $\Theta / E^{*}=1$ коэффициент восстановления всегда равен единице, поскольку в этом случае удар абсолютно упругий. На рис. 6 показаны зависимости коэффициентов восстановления для различных значений отношения $\Theta / E^{*}$. Нижняя кривая на рисунке построена при значении $\Theta / E^{*}=0$ и соответствует ситуации отсутствия упрочнения (эта кривая полностью совпадает с кривой, показанной звездочками на рис. 4). С увеличением значения коэффициента упрочнения, как это видно из рисунка, коэффициент восстановления $e_{p}$ возрастает. Это связано с тем, что с ростом параметра $\Theta / E^{*}$ диссипация механической энергии уменьшается. В предельном случае $\Theta / E^{*}=1$ мы всегда имеем значение $e_{p}=1$, поскольку в такой ситуации согласно рис. 5 реализуется только гуковский участок зависимости, а пластическая деформация при этом отсутствует. Таким образом, нами рассмотрен полный диапазон значений $0 \leq \Theta / E^{*} \leq 1$.

На вставке к рис. 6 показана зависимость коэффициента упрочнения от нормированной нормальной скорости для значения $\Theta / E^{*}=0.37$. Для такой ситуации существуют экспериментальные данные, полученные в работе [20] и теоретически описанные нами ранее в статье $[18]$ (показаны символами на вставке к рис. 6). Причем в [18] исследование приведено в размерных параметрах, при конкретном размере шарика, его плотности, фиксировался также параметр $q_{c}$. Здесь же для теоретического расчета зависимости необходимо знать только значения $\Theta / E^{*}$ и $v_{z 0}^{\text {crit }}$. Величина $v_{z 0}^{\text {crit }}$ взята нами непосредственно из работы [20] (это минимальное начальное значение нормальной скорости $v_{z}$, при которой начинаются пластические деформации). Параметр $\Theta / E^{*}$ в $[20]$ не приведен, поэтому в нашем рассмотрении он является подстроечным параметром. Помимо этого в [20] наблюдалось максимальное значение $e_{p}=0.95$, поэтому результаты эксперимента, показанные на вставке к рис. 6, нормированы на эту величину. В эксперименте значение $e_{p}=1$ не достижимо, поскольку помимо рассматриваемого механизма пластической деформации существуют другие каналы диссипации энергии, связанные с шероховатостями поверхностей, наличием загрязнений в области контакта, трением при движении шарика в воздушной среде, адгезией и т.д. Указанная нормировка позволяет исключить влияние этих эффектов. При этом существуют эксперименты, в которых коэффициент восстановления $e_{p}$ при малых скоростях даже больше 1, например [31] (см. также обзор [14]). Однако это практически всегда связано с неточностью проведения эксперимента, поскольку в описанных выше условиях такая ситуация соответствует нарушению закона сохранения энергии и не может быть реализована согласно первому закону термодинамики.

Дополнительно стоит отметить, что возможна также и ситуация, в которой $\Theta / E^{*}<0$, то есть коэффициент упрочнения $\Theta$ задает отрицательный наклон зависимости $F_{z}\left(u_{z}\right)$, показанной на рис. 5. При этом реализуется эффект разупрочнения, связанный с разрушением материала. Мы такой случай не рассматриваем, хотя применяемый нами метод позволяет провести моделирование при любой заданной зависимости $F_{z}\left(u_{z}\right)$. В случае разупрочнения и частичного разрушения материала коэффициент восстановления $e_{p}$ будет уменьшаться, поскольку появляется дополнительный канал диссипации энергии. Отметим также, что на рис. 6 пунктирной линией показано решение (24), полученное в работе [8]. Как видно, эта кривая сильно расходится с показанными на этом же рисунке экспериментальными результатами. Это лишний раз показывает, что теория, предложенная в [8], не описывает все возможные сценарии пластической деформации.

\section{5. Влияние адгезионного взаимодействия в зоне контакта}

На динамику соударений существенное влияние оказывает адгезионное взаимодействие, которое приводит к дополнительной диссипации механической энергии во время контакта. В теоретической работе [8] предложена модель, которая описывает одновременно наличие адгезионных сил и пластической деформации (см. также обзор [14]). В случае наличия одних лишь адгезионных сил (модель адгезионного взаимодействия JKR [32,33]) без 


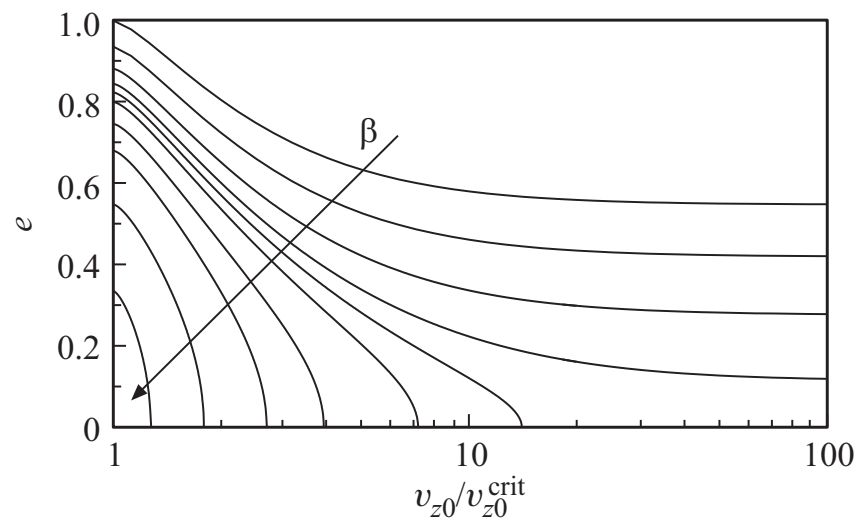

Рис. 7. Зависимости коэффициента восстановления нормальной скорости $e(30)$ как функции нормированного значения начальной скорости шарика $v_{z 0} / v_{z 0}^{\text {crit }}$, полученные при значении $\Theta / E^{*}=0.3$. Кривые, расположенные сверху вниз, построены при значении параметра $\beta=0,0.15,0.19,0.21,0.22,0.23$, $0.25,0.27,0.3,0.33$. Увеличение параметра $\beta$ дополнительно показано стрелкой.

пластической деформации коэффициент восстановления запишется как $[8,24]$

$$
e_{a}=-\frac{v_{z}}{v_{z 0}}=\sqrt{1-\frac{\sqrt[3]{4 \pi^{5}}}{10}(12+\sqrt[3]{2}) \beta^{5 / 2}},
$$

где коэффициент восстановления является функцией единственной переменной $\beta$, которая задается точным выражением [24]

$$
\beta=\left(\frac{R^{4} \Delta \gamma^{5}}{E^{* 2} m^{3} v_{z 0}^{6}}\right)^{2 / 15},
$$

где введена поверхностная энергия $\Delta \gamma$, а $m-$ это масса шарика, как и в (22). Авторы работы [8] для учета адгезии и пластических деформаций предлагают аппроксимацию

$$
e=\sqrt{e_{p}^{2}+e_{a}^{2}-1}
$$

где коэффициент восстановления $e_{p}$ в [8] задавался формулой (24), а в рассматриваемом нами случае нелокальной пластической деформации он показан на рис. 6. Используя формулы (28)-(30) и результаты моделирования, показанные на рис. 6 , легко рассчитать значение $e(30)$ в общем случае адгезионного контакта с наличием пластической деформации и эффекта упрочнения. Стоит отметить, что аппроксимация (30) является приближением, которое описывает влияние адгезии скорее качественным образом, и для точного выяснения такого влияния необходимо проводить дополнительное моделирование. Однако для определения основных закономерностей поведения рассматриваемой системы использования формулы (30) вполне достаточно.
На рис. 7 показаны рассчитанные по формуле (30) зависимости коэффициента восстановления $e$ от безразмерной скорости $v_{z 0} / \nu_{z 0}^{\text {crit }}$, где $v_{z 0}^{\text {crit }}$ определялась введенным выше соотношением (25). Здесь верхняя кривая $(\beta=0)$ повторяет кривую при $\Theta / E^{*}=0.3$, приведенную на рис. 6 (там это четвертая кривая снизу). Для $\beta=0$ адгезия отсутствует и диссипация механической энергии осуществляется только за счет пластической деформации. Согласно рисунку с увеличением параметра $\beta$ коэффициент восстановления уменьшается, поскольку вклад адгезии в общую диссипацию энергии увеличивается. Поскольку на рис. 6 значение $v_{z 0} / v_{z 0}^{\text {crit }}=1$ соответствует скорости, при которой начинается пластическая деформация, в этой точке диссипация происходит только за счет адгезии. Видно, что с ростом параметра $\beta$ коэффициент восстановления $e$ при $v_{z 0} / \nu_{z 0}^{\text {crit }}=1$ также уменьшается. Из формулы (28) легко определить значение $\beta$, при котором вся механическая энергия будет затрачена на работу сил адгезии, в отсутствие пластических деформаций. Это значение получается из (28) при $e_{a}=0$, что дает

$$
\beta_{c}=\frac{5^{2 / 5} 2^{2 / 15}}{\left(12+2^{1 / 3}\right)^{2 / 5} \pi^{2 / 3}} \approx 0.3461590663 .
$$

При $\beta>\beta_{c}$ (31) частица не отражается от поверхности полупространства, а прилипает к ней за счет адгезии.

На рис. 7 нами показаны зависимости коэффициента восстановления $e$ как функции безразмерной скорости $v_{z 0} / \nu_{z 0}^{\text {crit }}$ и параметра $\beta(29)$. Однако, в работе [8] авторы предлагают другую на наш взгляд интересную параметризацию такой задачи. Они приводят аналогичные зависимости, только фиксируют не параметр $\beta$, а другой безразмерный параметр, который равен отношению критической скорости, при которой за счет адгезионных сил происходит полная диссипация механической энергии $v_{z 0}^{*}$, к ранее введенной величине $v_{z 0}^{\text {crit }}$. Скорость $v_{z 0}^{*}$ может быть найдена из выражений (31) и (29), что приводит к соотношению вида [8]

$$
v_{z 0}^{*}=\sqrt{\frac{3\left(12+2^{1 / 3}\right)}{20}}\left(\frac{\pi^{2} \Delta \gamma^{5}}{2 E^{* 2} R^{5} \rho^{3}}\right)^{1 / 6} .
$$

В случае $v_{z 0}<v_{z 0}^{*}$ частица за счет адгезии остается на поверхности и не отражается от нее, даже в случае отсутствия пластических деформаций.

На рис. 7 все кривые начинаются со значения коэффициента восстановления $e$, которое не изменяется при уменьшении скорости ниже $v_{z 0} / \nu_{z 0}^{\text {crit }}$, поскольку для таких скоростей пластическая деформация отсутствует. Как мы указывали выше, в работе [8] фиксируется значение безразмерного параметра $v_{z 0}^{*} / \nu_{z 0}^{\text {crit }}$, а не параметра $\beta$. Такое представление результатов может давать в некотором смысле более наглядную картину. Зависимости коэффициента восстановления от значения $v_{z 0} / v_{z 0}^{\text {crit }}$ при фиксированных величинах $v_{z 0}^{*} / v_{z 0}^{\text {crit }}$ могут быть рассчитаны используя результаты, показанные на рис. 6. Для этого необходимо использовать формулу для 


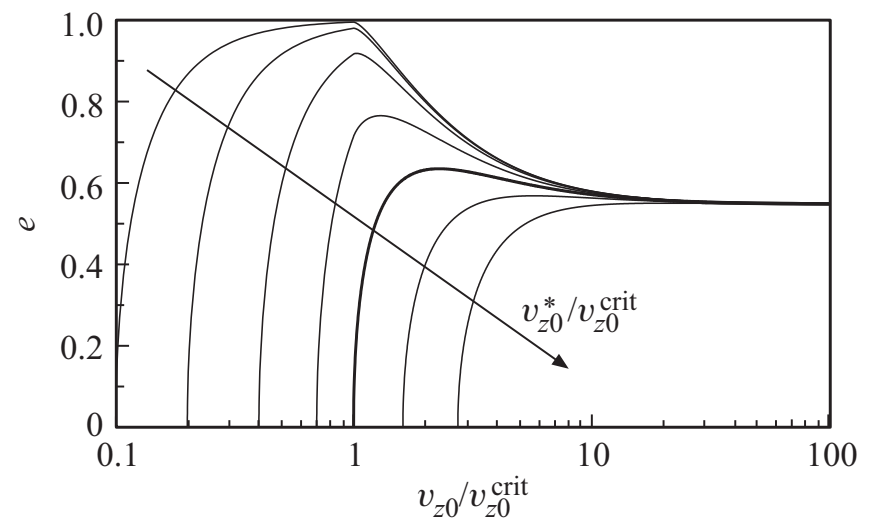

Рис. 8. Зависимости коэффициента восстановления нормальной скорости $e(30)$ как функции нормированного значения начальной скорости шарика $\nu_{z 0} / \nu_{z 0}^{\text {crit }}$, при значении $\Theta / E^{*}=0.3$. Кривые, расположенные сверху вниз, построены при значении параметра $v_{z 0}^{*} / v_{z 0}^{\text {crit }}=0.1,0.2,0.4,0.7,1.0,1.4,2.0$. Увеличение параметра $v_{z 0}^{*} / v_{z 0}^{\text {crit }}$ дополнительно показано стрелкой.

коэффициента восстановления при диссипации механической энергии за счет сил адгезии (28), которую можно записать также в ином виде [8]

$$
e_{a}=\sqrt{1-\left(\frac{v_{z 0}^{*}}{v_{z 0}}\right)^{2}} .
$$

Перепишем последнее выражение как

$$
e_{a}=\sqrt{1-\left(\frac{v_{z 0}}{v_{z 0}^{\text {crit }}}\right)^{-2}\left(\frac{v_{z 0}^{*}}{v_{z 0}^{\text {crit }}}\right)^{2}} .
$$

Запись (34) является полным эквивалентом (33), однако выражение в такой форме позволяет используя результаты, представленные на рис. 6, вычислить значение $e_{a}$, соответствующее набору фиксированных отношений $v_{z 0} / \nu_{z 0}^{\text {crit }}$ и $v_{z 0}^{*} / \nu_{z 0}^{\text {crit. }}$. Далее, используя аппроксимацию (30), легко можно найти значение коэффициента восстановления $e$. Рассчитанные таким образом зависимости показаны на рис. 8. Из рисунка следует, что с увеличением значения безразмерного параметра $v_{z 0}^{*} / \nu_{z 0}^{\text {crit }}$ коэффициент восстановления $e$ также уменьшается, за счет увеличения вклада адгезии в общую диссипацию механической энергии. Пластическая деформация здесь как и на предыдущих рисунках начинается после значения скорости $v_{z 0} / v_{z 0}^{\text {crit }}>1$, поэтому до этого значения потери энергии осуществляются только за счет адгезии, а коэффициент восстановления определяется формулой (34). Для кривой, построенной при $v_{z 0}^{*} / v_{z 0}^{\text {crit }}=1$ (пятая кривая сверху, показана жирной линией на рисунке) выполняется соотношение $v_{z 0}^{\text {crit }}=v_{z 0}^{*}$, т.е. при значении скорости, при которой начинаются пластические деформации, вся энергия диссипируется за счет адгезии, поэтому в этой точке реализуется величина $e=0$. С дальнейшим увеличением начальной скорости $v_{z 0}$ вклад в диссипацию энергии за счет адгезии уменьшается, а коэффициент восстановления нарастает, несмотря на то, что вклад пластических деформаций с увеличением скорости в общую диссипацию энергии при этом возрастает (см. рис. 6 и 7). Результаты, показанные на рис. 8, качественно повторяют аналогичные результаты, полученные в работе [8]. Однако в нашем случае имеются существенные количественные отличия, поскольку мы рассмотрели случай нелокальной пластичности, задающийся критерием (10) и провели учет эффекта деформационного упрочнения, отсутствующего в [8].

\section{6. Заключение}

В работе предложена численная модель, описывающая нормальное столкновение сферических тел, с наличием пластических деформаций и сил адгезии в зоне контакта. Выяснено, от каких параметров зависит значение коэффициента восстановления нормальной скорости. Соответствующие параметры записаны в безразмерном виде и в широком диапазоне таких параметров проведено численное моделирование процесса столкновения. Интересно то, что полученные результаты с высокой точностью совпадают с экспериментальными результатами из работы [20]. Классические теории такого совпадения не показывают. Более того, нами был проведен учет деформационного упрочнения, которое практически всегда имеет место при пластических деформациях. Это является преимуществом по сравнению с классическими моделями теории столкновений. Показано, что коэффициент восстановления зависит от трех безразмерных параметров, которые зависят от начальной скорости, а также от геометрических, материальных и адгезионных характеристик контактирующих материалов. Полученное в работе семейство кривых способно описать эксперименты с различным набором параметров, которые определяют значения указанных безразмерных величин. Предложенное описание справедливо при реализации условий нелокальной пластичности, в области, где отсутствует канал диссипации энергии, связанный с разрушением и растрескиванием образца, то есть где реализуется пластическая область кривой нагружения.

Автор выражает благодарность В.Л. Попову за полезные обсуждения в процессе проведения исследования.

\section{Список литературы}

[1] M.P. Ciamarra, A.H. Lara, A.T. Lee, D.I. Goldman, I. Vishik, H.L. Swinney. Phys. Rev. Lett. 92, 194301 (2004).

[2] P. Jop, Y. Forterre, O. Pouliquen. Nature 441, 727 (2006).

[3] N.V. Brilliantov, F. Spahn, J.-M. Hertzsch, T. Pöschel. Phys. Rev. E 53, 5382 (1996).

[4] F.G. Bridges, A. Hatzes, D.N.C. Lin. Nature 309, 333 (1984).

[5] N.V. Prodanov, A.V. Khomenko. Surf. Sci. 604, 730 (2010).

[6] I.A. Lyashenko. FU Mech. Eng. 14, 313 (2016).

[7] В.В. Ефименко. Вестн. ФГОУ ВПО МГАУ 2, 41 (2009).

[8] C. Thornton, Z. Ning. Powder Technol. 99, 154 (1998). 
[9] P. Müller, R. Böttcher, A. Russell, M. Trüe, S. Aman, J. Tomas. Adv. Powder Technol. 27, 1233 (2016).

[10] P. Müller, R. Böttcher, A. Russell, M. Trüe, J. Tomas. Chem. Eng. Sci. 138, 689 (2015).

[11] S. Aman, P. Müller, J. Tomas, S. Kozhar, M. Dosta, S. Heinrich, S. Antonyuk. Adv. Powder Technol. 27, 1244 (2016).

[12] L.-Y. Li, C. Thornton, C.-Y. Wu. Proc. Instn. Mech. Eng. Part C 214, 1107 (2000).

[13] J. Xie, M. Dong, S. Li. Aerosol Sci. Technol. 50, 321 (2016).

[14] Я.А. Ляшенко, Э. Виллерт, В.Л. Попов. Вестн. ПНИПУ. Механика 2, 44 (2018).

[15] M.R.W. Brake. Int. J. Solids Struct. 62, 104 (2015).

[16] J. Ma, D. Liu, X. Chen. Aerosol Air Qual. Res. 16, 1533 (2016).

[17] T. Müller, F. Gollwitzer. AIP Conf. Proc. 1542, 787 (2013).

[18] Я.А. Ляшенко, В.Л. Попов. ФТТ 60, 560 (2018).

[19] V.L. Popov. FU Mech. Eng. 13, 39 (2015).

[20] J.M. Lifshitz, H. Kolsky. J. Mech. Phys. Solids 12, 35 (1964).

[21] V.L. Popov, M. Heß. Method of dimensionality reduction in contact mechanics and friction. Springer-Verlag, Berlin (2015). 265 p.

[22] I.A. Lyashenko, V.L. Popov. Sci. Rep. 5, 8479 (2015).

[23] E. Willert, V.L. Popov. Z. Angew. Math. Mech. 96, 1089 (2016).

[24] I.A. Lyashenko, E. Willert, V.L. Popov. Mech. Mater. 92, 155 (2016).

[25] V.L. Popov, E. Willert, M. Heß. FU Mech. Eng. 16, 99 (2018).

[26] H.J. Hertz. J. Reine Angew. Math. 92, 156 (1882).

[27] R.M. Davies. Proc. R. Soc. London A 197, 416 (1949).

[28] H. Minamoto, S. Kawamura. Int. J. Impact Eng. 38, 123 (2011).

[29] A.H. Kharaz, D.A. Gorham. Philos. Mag. Lett. 80, 549 (2000).

[30] А.Д. Погребняк, С.Н. Братушка, В.M. Береснев, N. Levintant-Zayonts. Успехи химии 82, 1135 (2013).

[31] C.M. Sorace, M.Y. Louge, M.D. Crozier. Mech. Res. Commun. 36, 364 (2009).

[32] K.L. Johnson, K. Kendall, A.D. Roberts. Proc. R. Soc. London A 324, 301 (1971).

[33] A. Papangelo. FU Mech. Eng. 16, 19 (2018).

Редактор Т.Н. Василевская 